\title{
Project 1021951
}

\section{In-Situ Survival Mechanisms of U and Tc Reducing Bacteria in Contaminated Sediments}

\author{
Krumholz, Lee R. \\ University of Oklahoma
}

RESULTS TO DATE: Summary: Desulfovibrio desulfuricans G20 and Shewanella oneidensis MR-1 are model subsurface organisms for studying genes involving in situ radionuclide transformation and sediment survival. Our research objective for this project has been to develop a signature-tagged mutagenesis (STM) procedure and use it to identify mutants in genes of these subsurface bacteria involved in sediment survival and radionuclide reduction. The mutant genes identified in these studies allow us for the first time to describe at the genetic level microbial processes that are actually being used by environmental bacteria while growing in their natural ecosystems. Identification of these genes revealed facets of microbial physiology and ecology that are not accessible through laboratory studies. Ultimately, this information may be used to optimize bioremediation or other engineered microbial processes. Furthermore, the identification of a mutant in a gene conferring multidrug resistance in strain MR-1 shows that this widespread mechanism of antibiotic resistance, likely has its origins as a mechanism of bacterial defense against naturally occurring toxins. Studies with D. desulfuricans G20: The STM procedure first involved generating a library of 5760 G20 mutants and screening for potential non-survivors in subsurface sediment microcosms. After two rounds of screening, a total of 117 mutants were confirmed to be true non-survivors. 97 transposon insertion regions have been sequenced to date. Upon further analysis of these mutants, we classified the sediment survival genes into COG functional categories. STM mutant insertions were located in genes encoding proteins related to metabolism (33\%), cellular processes (42\%), and information storage and processing (17\%). We also noted $8 \%$ of STM mutants identified had insertions in genes for hypothetical proteins or unknown functions. Interestingly, at least 64 of these genes encode cytoplasmic proteins, 46 encode inner membrane proteins, and only 7 encode periplasmic space and outer membrane associated proteins. Through blast search analysis, we also showed that 81 out of 94 proteins shown to be important in sediment survival have homologs in D. vulgaris, 70 have homologs in Geobacter metallireducens, and 69 have homologs in Geobacter sulfurreducens PCA. Some interesting proteins include ribonucleotide reductase and chemotaxis related proteins. Ribonucleotide reductase catalyzes the reductive synthesis of deoxyribonucleotides from their corresponding ribonucleotides, providing the precursors necessary for DNA synthesis. Two ribonucleotide reductase genes (nrdE, nrdD) were found to be essential for G20 survival in the sediment, but not essential for growth in the lactate-sulfate medium. Bacterial methyl-accepting chemotaxis proteins (MCP) respond to changes in the concentration of attractants and repellents in the environment. Two out of 30 MCPs of G20 were identified to be crucial for sediment survival. Another chemotaxis protein stimulating methylation of MCP was also found to be important for sediment survival, although it does not form an operon with either of the two MCP?s identified in our STM screen. Three histidine kinase defective mutants lost the ability to survive in sediment. They may form part of two-component regulatory systems with chemotaxis proteins or regulate expression in other systems. Many more genes have been characterized which are required for sediment survival. Our analyses will likely allow us to propose a detailed model for the interactions and physiological processes associated with radionuclide-reducing bacteria as they live and grow in aquifer systems. We have also screened the STM library for mutants unable to transform $\mathrm{U}(\mathrm{VI})$. To date, nine mutants have been identified which are impaired in the ability to grow in lactate-sulfate medium with $2 \mathrm{mM} \mathrm{U}(\mathrm{VI})$-acetate. Six of these mutants are completely unable to grow. Transposon insertion sites have been amplified, sequenced, and studied at the bioinformatics level, revealing that several genes disrupted may be involved in DNA, RNA and protein maintenance. We have identified three genes believed to be directly involved in DNA repair, one gene involved in rRNA methylation, which could stabilize rRNA under stress, and one RNA polymerase subunit which is required for RNA polymerase renaturation. Several other mutated genes may be involved in redox reactions within the cell, as two mutations observed might be involved in molybdopterin synthesis and one mutation was in a hypothetical protein with a thioredoxin domain. Orthologs to this hypothetical protein are present in D. vulgaris, Geobacter metallireducens and G. sulfurreducens. We also obtained mutants in genes for a 
phosphotransacetylase, a dnaJ-like gene and a histidine kinase. The latter two genes are known to be involved in stress responses. Based on the analysis of mutants, uranium appears to exert its toxicity at DNA, RNA and protein levels. Resistance to the effects of $\mathrm{U}(\mathrm{VI})$ involve either detoxification, which likely involves biological reduction, or repair of damaged cell material. S. oneidensis: The ability of $\mathrm{S}$. oneidensis MR-1 to reduce radionuclides, including technetium and uranium, has generated great interest in its use for bioremediation. Optimization of in situ bioremediation processes require knowledge of the interaction between MR-1 and its natural environment. We are currently using STM to examine this interaction at the genetic level in a similar manner to the studies discussed above with strain G20. To date, STM has revealed a number of genes critical for survival in sediments. Of approximately 5000 MR-1 transposon mutants screened in sediments, 48 genes representing general cellular processes of DNA repair, transport, transcriptional regulation, energy and amino acid metabolism, as well as genes encoding phage related and transposon related proteins were identified as critical to sediment survival. One gene of particular interest is the mexF, identified as a gene involved in mutidrug resistance. Further characterization of this mutant strain has been carried out. Growth was examined in the presence of various antibiotics under a range of aerobic and anaerobic conditions. While no difference was found between the mexF mutant and parental strain for several antibiotics, mutant growth was impaired for tetracycline and chloramphenicol concentrations tested. Other STM mutants with transposon insertions in genes with potential detoxification functions were also tested on tetracycline and chloramphenicol, with results that were similar to the parental strain. The MexF protein has been characterized in a pathogenic Pseudomonas strain and when overexpressed, will protect the cells from exposure to antibiotics. We propose that MR-1 mexF, whose encoded protein product shares $70 \%$ identity across the amino acid sequence to previously characterized Pseudomonas MexF, functions in detoxification of ambient concentrations of naturally occurring antimicrobials encountered by this environmental bacterium.

DELIVERABLES: Publications: 1. Groh, J.L., Q. Luo, J.D. Ballard and L.R. Krumholz. Adaptation of microarray technology for Signature Tagged Mutagenesis (STM) of Desulfovibrio desulfuricans G20 and Shewanella oneidensis MR-1 in anaerobic sediment survival experiments. Appl. Env. Microbiol. (in press).

2. Chang, I.S., J. L. Steger, M. M. Ramsey, J.D. Ballard and L.R. Krumholz. 2004. Differential Expression of Desulfovibrio vulgaris genes in response to $\mathrm{Cu}(\mathrm{II})$ and $\mathrm{Hg}(\mathrm{II})$ toxicity. Appl. Environ. Microbiol. 70:18471851.

3. Elias, D.A., J.M. Suflita, M.J. McInerney and L.R. Krumholz. 2004. The periplasmic cytochrome C3 of Desulfovibrio vulgaris is directly involved in $\mathrm{H} 2$ mediated metal-, but not sulfate-reduction. Appl. Environ. Microbiol. 70(1):413-420.

4. Chang, I., J.D. Ballard and L.R. Krumholz. 2003. Evidence for Chimeric sequences during random arbitrarily primed PCR. J. Microbiol Meth. 54:427-431.

5. Steger, J.L., C. Vincent, J.D. Ballard and L.R. Krumholz. 2002. Desulfovibrio sp. Genes involved in the metabolism of Hydrogen and Lactate. Appl. Environ. Microbiol. 68(4):1932-1937.

\section{Abstracts:}

Groh, J.L. and L. R. Krumholz. 2005. Multidrug Resistance Function of MexF is Necessary for Sediment Survival of Shewanella oneidensis MR-1. The 105th general meeting of the American Society for Microbiology. Atlanta, GA . Q-274.

Li, X., Q. Luo and L. R. Krumholz. 2005. Identification of Desulfovibrio desulfuricans G20 Genes Required for Resistance to Uranium. The 105th general meeting of the American Society for Microbiology. Atlanta, GA . Q-058. 
Qingwei Luo, Jennifer L. Groh, Jimmy D. Ballard and Lee R. Krumholz. 2004. Signature tagged mutagenesis (STM) for identification of genes required for in situ sediment survival of Desulfovibrio strain G20 and Shewanella oneidensis MR-1. Abstract of the NABIR PI meeting, Warrenton, VA

Jennifer L. Groh and Lee R. Krumholz. 2004. Growth of Shewanella oneidensis MR-1 in sediment microcosms for identification of sediment survival genes. Abstract of the NABIR PI meeting, Warrenton, VA.

JENNIFER L. GROH* AND LEE R. KRUMHOLZ. 2004. Signature tagged mutagenesis (STM) for identification of genes required for in situ sediment survival of Shewanella oneidensis MR-1. Abstract of the Missouri Valley Regional ASM meeting. Kansas City, MO.

QINGWEI LUO *, and LEE R. KRUMHOLZ. 2004. Identification of Genes Required for In Situ Sediment Survival of Desulfovibrio desulfuricans Strain G20 by Signature Tagged Mutagenesis (STM). Abstract of the Missouri Valley Regional ASM meeting. Kansas City, MO.

NYDIA CASTANEDA*, MARVIN WHITELEY, LEE KRUMHOLZ. 2004. Isolation and Characterization of a Plasmid from Desulfovibrio africanus SR-1. Abstract of the Missouri Valley Regional ASM meeting. Kansas City, MO.

X. Li and L. Krumholz. 2004. . As(V) Reduction by Desulfovibrio Strains and Dual As(V) Resistance System of Desulfovibrio desulfuricans G20. Abstracts of the ASM annual meeting. New Orleans, LA Q013.

I. N. Castaneda-Carrion, M. Whiteley and L. R. Krumholz. 2004. Isolation and Characterization of a Plasmid from Desulfovibrio africanus SR-1. Abstracts of the ASM annual meeting. New Orleans, LA H125.

Jennifer L. Groh, Qingwei Luo, Jimmy D. Ballard and Lee R. Krumholz. 2004. Signature tagged mutagenesis (STM) for identification of genes required for in situ sediment survival of Shewanella oneidensis MR-1. Abstracts of the ASM annual meeting. New Orleans, LA

Qingwei Luo, Jennifer L. Groh, Xiangkai Li, Jimmy D. Ballard and Lee R. Krumholz. 2004. Signature Tagged Mutagenesis (STM) for Identification Genes Required for In Situ Sediment Survival of Desulfovibrio Strain G20. Abstracts of the ASM annual meeting. New Orleans, LA

Qingwei Luo, Jennifer L. Steger, Darren J. Smalley, Jimmy D. Ballard and Lee R. Krumholz. 2003. A new screening system with microarray technology for signature tagged mutagenesis with Desulfovibrio and Shewanella. Abstracts of the ASM annual meeting. Washington, DC. 